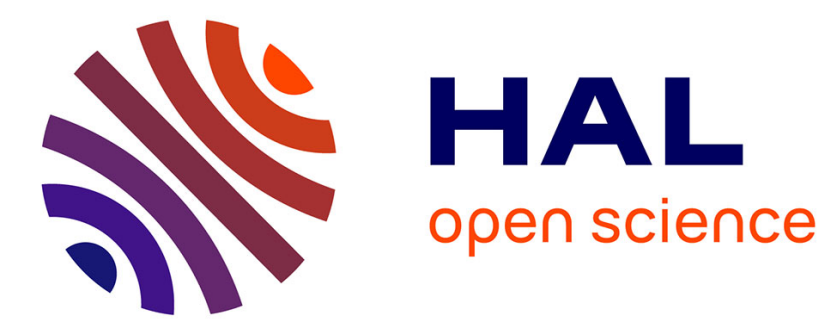

\title{
Inquiry, Refutations and the Inconsistent
}

Can Baskent

\section{- To cite this version:}

| Can Baskent. Inquiry, Refutations and the Inconsistent. 2014. hal-01094783

\section{HAL Id: hal-01094783 \\ https://inria.hal.science/hal-01094783}

Preprint submitted on 13 Dec 2014

HAL is a multi-disciplinary open access archive for the deposit and dissemination of scientific research documents, whether they are published or not. The documents may come from teaching and research institutions in France or abroad, or from public or private research centers.
L'archive ouverte pluridisciplinaire HAL, est destinée au dépôt et à la diffusion de documents scientifiques de niveau recherche, publiés ou non, émanant des établissements d'enseignement et de recherche français ou étrangers, des laboratoires publics ou privés. 


\title{
Inquiry, Refutations and the Inconsistent
}

\author{
Can Başkent \\ INRIA, Nancy \\ www.canbaskent.net/logic, can@canbaskent.net
}

May 29, 2014

\begin{abstract}
In this paper, I discuss the connection between Lakatosian method of proofs and refutations, Hintikkan models of interrogative inquiry and paraconsistency. I bridge these different schools with dialectic, and their underlying reliance on the inconsistent.

Key words Lakatos's proofs and refutations, Hintikka's interrogative models of inquiry, paraconsistency.
\end{abstract}

\section{Introduction}

In this paper, I argue that Lakatos's methodology of scientific research programs as exemplified in Proofs and Refutations and Hintikka's interrogative models of inquiry share various epistemic and logical qualities. I furthermore claim that paraconsistency is one of such qualitative similarities between the Lakatosian and the Hintikkan research programs even though neither of the philosophers was explicitly committed to this view 1

The organization of this paper is as follows. First, I discuss the epistemic and methodological similarities between Hintikka's inquiry and Lakatos's research program. Then, I analyze those similarities from the view point of inconsistencytolerant, paraconsistent logical approach.

What I claim here does not reject Lakatos's or Hintikka's results, but it questions the choice of underlying logic (which is the classical logic) which they used in their frameworks. My arguments unearth the hidden logical commitments of both philosophers, which I think is evident in their works but not widely discussed. I am not directly arguing that both philosophers favor inconsistencytolerant logics. Instead, I claim that their methodological frameworks make me question their commitment to classical logic, and that their systems have some aspects that intrinsically admit paraconsistency.

\footnotetext{
${ }^{1}$ It is important to note that Hintikka recently made some suggestions to combine IF logic -which does not entirely fall within the scope of this paper- with paraconsistent logics (Hintikka, 2009 Carnielli, 2009).
} 
I now start with reviewing Lakatos's and Hintikka's frameworks from inconsistency - tolerant point of view.

\section{Hintikka and Lakatos}

Hintikka's model of interrogative inquiry is a well-known example of a dynamic epistemic procedure that results in knowledge increase. Simply put, in an interrogative inquiry, the inquirer is given a theory and a question. He then tries to answer the question based on the theory by posing some questions to nature or an oracle. In an interrogative inquiry, the inquirer has two options. He is allowed to ask questions to nature/oracle, conceived as a truthful source of information, or alternatively draw conclusions by using the given base theory and the answers he has already received.

The interrogative models of inquiry has largely been studied by the Helsinki School, and the major arguments of this research program can be found in a series of articles (Hintikka, 1988; Hintikka, 1984; Hintikka, 1987; Hintikka \& Harris, 1988; Hintikka et al., 1999; Halonen \& Hintikka, 2005; Hintikka, 2007; Garrison, 1988; Genot, 2009). Recently, Carnielli studied the connection between interrogative models and paraconsistency, which also influenced the current paper (Carnielli, 2009). Carnielli, after Hintikka's recent sympathy towards paraconsistency (Hintikka, 2009), remarks that "the problem of coping with contradictory information belongs to interrogative games", which seems to agree with our perspective in this work (Carnielli, 2009).

The procedure that interrogative inquires follows is simple. Yet, it admits some hidden assumptions that are not widely discussed. The first hidden assumption of Hintikkan inquiry is its reliance on classical logic and its rules of derivation. However, the epistemic procedure of interrogative inquiry does not require such a commitment to classical logic by- and in-itself.

In order to illustrate our argument, consider the following aspect of inquiry. In inquiry, players are allowed to bracket out some answers to eliminate them from the procedure if they think those answers are not relevant or do violate the consistency of the system. Hintikka writes:

An important aspect of this general applicability of the interrogative model is its ability to handle uncertain answers - that is, answers that may be false. The model can be extended to this case simply by allowing the inquirer to tentatively disregard ("bracket") answers that are dubious. The decision as to when the inquirer should do so is understood as a strategic problem, not as a part of the definition of the questioning game. Of course, all the subsequent answers that depend on the bracketed one must then also be bracketed, together with their logical consequences. Equally obviously, further inquiry might lead the inquirer to reinstate ("unbracket") a previously bracketed answer. This means thinking of interrogative inquiry as a self-corrective process. It likewise means considering discovery and 
justification as aspects of one and the same process. This is certainly in keeping with scientific and epistemological practice. There is no reason to think that the interrogative model does not offer a framework also for the study of this self-correcting character of inquiry.

$$
\text { (Hintikka, 1962, p. 3) }
$$

In an earlier paper, I focused on the epistemological redundancy of bracketing in Hintikkan inquiry where I argued that the existence of inconsistencies is natural (and even desirable) in a dialogical inquiry. Yet, we can still make meaningful deductions under the presence of inconsistencies rendering the working system a paraconsistent one (Başkent, 2014). Other problems of the bracketing in Hintikkan inquiry include epistemic, game theoretical and heuristic problems where the heuristic issues are quite central also for Lakatos.

Epistemically, there seems to be a major problem in bracketing. In an inquiry or a dialogue game, how can we know which answers to ignore beforehand? How can we know what to reject or accept? This epistemic problem empties the notion of bracketing. In other words, if inquiry is a procedure during which we want to acquire and learn some information, this implies that we did not have that information before. In an epistemic inquiry, we are supposed to be searching and looking for some information that we did not have before. We cannot discard some responses in favor of or against some questions or propositions simply because we do not know the answer. If we knew, we would not ask.

A game theoretical response can be given to eliminate this problem, arguing against my point. Namely, in an inquiry, we simply choose the assumptions and responses that help us win the game. If we can win the game with a particular set of assumptions, then we adopt these assumptions as they give us a win. If we fail to win the game with that particular set of assumptions and the previous answers we received in the inquiry, we simply select another set of assumptions and answers, and keep playing, and repeat the procedure if necessary.

However, this objection undermines the agency of the players. In a game theoretical setting, each player follows a strategy to choose their moves. By definition, a strategy is predetermined and preset before the game based on some understanding of rationality and players' priors (and perhaps some probabilistic calculus). Borrowing the concepts of traditional game theory, therefore, a player's strategy considers all possible ways of plays for the opponent, and includes ways to respond to them (to counter-act the possible attacks). A strategy is pre-determined, and fully inclusive of all the possibilities - at least theoretically. An unexpected move of the opponent, a new piece of information and its consequences and many other possibilities, should therefore be already included in the strategy, by definition. Players decide, and set their strategy, and determine how they will play before they start playing the game. If we allow them to exercise their choice of moves based on their a posteriori success, that means that they did not have an a priori strategy before the game-play. Simply put, a game theoretical player is rational, and constructs a strategy based on his priors, as opposed to deciding how to play during the game. Therefore, such an objection clashes with the basic definition of a strategy - a function that tells 
the player which move to make at each state based on what moves the other players have made (Başkent, 2011).

Finally, bracketing suffers from various central problems from a heuristic point of view. First, let us remember the Lakatosian concept of "proofs that do not prove" which is directly relevant and helpful to our investigation. Lakatosian methodology of proofs and refutations, as exemplified in Proofs and Refutations for instance, discusses the significant roles of (unsuccessful) thought experiments, informal proofs and unsound deductions in mathematical reasoning among many other things (Lakatos, 2005; Lakatos, 1979; Başkent \& Bag̃çe, 2009). "Proofs that do not prove" are the proofs that are wrong in some ways, yet help us develop better proofs or improve the current false proof. Lakatos discusses this idea in detail, and explains its role in concept formation with many historical examples. For Lakatosian epistemology, in an evolutionary and practice based sense, mathematical concepts develop, improve and then they are falsified, proven and disproven along their conceptual development. Mathematical activity continues, and the concepts are redeveloped, the proofs are re-examined. In short, "proof attempts" help us improve the proofs. However, if we bracket "proofs that do not prove", we risk the growth of (mathematical) knowledge, and lose the opportunity to learn from our mistakes (Başkent, 2014).

I already discussed the above points in an earlier work (Başkent, 2014). Now, my focus is the self-correcting character of inquiry which bears some similarities to Lakatosian methods that include informal proofs, thought experiments and quasi-emprical view of mathematical activity, bridging the two as we shall see.

In my understanding, what Hintikka alludes in the above lengthy quote is that a scientific theory revises itself to exclude inconsistencies or incoherencies, and interrogative inquiry, as a special case of this phenomenon, follows a similar procedure. In Hintikka's perspective, this is the point that prevents him from being a pluralist logician - disallowing multiple conclusions in the deductive relation of the logic he uses. In short, whenever there seems to be a problem within the theory, the theory utilizes its own internal tools to fix itself. Some call it belief revision, some call it epistemic updates, there are various other logical methods which operate with a similar method to achieve a similar goal (Genot, 2009; Garrison, 1988).

However, note that this procedure itself is paraconsistent even though it aims at preserving the consistency at the end. Recall that paraconsistency is the umbrella term for the logical systems where inconsistencies do not trivialize the system. In paraconsistent systems, we can have $\varphi, \neg \varphi \nvdash \psi$ for some $\varphi, \psi$. Dialogues can be thought of an example of paraconsistent phenomena (Rahman $\&$ Carnielli, 2000; Rahman \& Tulenheimo, 2009). A careful approach to terminology is in order here. Paraconsistency is usually confused with dialetheism which is the view that suggests that some contradictions are true. Paraconsistency is a rather proof-theoretical approach whereas dialetheism is a semantical one. Additionally, it would be wise to underline the fact that logicians often distinguish contradictions from the inconsistent (Carnielli et al., 2007). For the purposes of 
this paper, we will assume that contradictions create inconsistencies. We will not suggest that every inconsistency is caused by a contradiction. Moreover, for the technically oriented reader, as they will realize throughout the paper, we will refrain ourselves from explicitly committing to a specific form of paraconsistent logic. Paraconsistent logics form a broad spectrum of logical formalisms motivated by various philosophical insights, and produce relatively different mathematical results. It should be clear that our philosophical treatment of the subject, at this stage, does not necessarily require any explicit commitment to a specific branch or understanding of paraconsistent school of logic, and more importantly we are, at least currently, not suggesting a paraconsistent logic for Hintikkan inquiry or Lakatosian method of proofs and refutations.

For Hintikka, an inquiry, in its broadest generality, can have some inconsistent statements which might have arisen from the dialogue or inquiry, yet, we must not include them in our deductive process. However, this means that, under the presence of inconsistencies, we still make some meaningful deduction - even if this deduction attempts at excluding those very inconsistencies and contradictions. We will perhaps ignore inconsistencies epistemologically, yet, logically they are simply there in the form of a set of contradictory answers perhaps. There can be thought of various choice mechanisms that determine which propositions and responses we need to include or exclude from the deductive process of the inquiry. Moreover, the decidability of the logical system (if it is first-order or propositional) makes a distinctive difference whether we can determine which responses to include or exclude from the procedure in order to maintain a coherent and consistent system. Yet, aside from the computational aspects of it and its difficulties, the very decision of bracketing some of contradictory statements is taken under the very existence of the same contradictory statements. This is a working paraconsistent procedure.

The crucial point here, as I underlined earlier, is that Hintikka thinks that the system will eventually correct itself. For him, after some thought-experiments or quasi-emprical observations, we will reach the true statements with an inquiry even if we may have hit some inconsistencies along the way. Here, again, notice that the very existence of the inconsistencies along the way does not trivialize the model. Hintikka does not seem to enjoy epistemic inconsistencies, yet he does not logically exclude them from his system in a convincing way.

A very similar issue appears in Lakatosian methodology as well. First, let us briefly recall Lakatosian method of proofs and refutations. Lakatosian methodology follows a simple yet well-defined road map which consists of the following methodological steps which I borrow from Corfield (Corfield, 1997):

1. Primitive conjecture.

2. Proof (a rough thought experiment or argument, decomposing the primitive conjecture into subconjectures and lemmas).

3. Global counterexamples.

4. Proof re-examined. The guilty lemma is spotted. The guilty lemma may have previously remained hidden or may have been misidentified. 
5. Proofs of the other theorems are examined to see if the newly found lemma occurs in them.

6. Hitherto accepted consequences of the original and now refuted conjecture are checked.

7. Counterexamples are turned into new examples, and new fields of inquiry open up.

As the above account identifies, Lakatos's method of proofs and refutations is a quite systematic account of mathematical discovery with a strong emphasis on mathematical practice. There are various strong criticisms towards Lakatos from mathematical angles, yet I will now dwell into them in this paper (Koetsier, 1991).

One of my favorite passages of Proofs and Refutations discusses the Cauchian revolution of rigor in mathematics versus axiomatic Euclidean methodology.

The Cauchy revolution of rigour was motivated by a conscious attempt to apply Euclidean methodology to the Calculus. He and his followers thought that this was how they could introduce light to dispel the 'tremendous obscurity of analysis'. Cauchy proceeded in the spirit of Pascal's rules: he first set out to define the obscure terms of analysis - like limit, convergence, continuity etc. - in the perfectly familiar terms of arithmetic, and then he went on to prove everything that had not previously been proved, or that was not perfectly obvious. Now in the Euclidean framework there is no point trying to prove what is false (My emphasis), so Cauchy had first to improve the extant body of mathematical conjectures by jettisoning the false rubbish. (...) What was considered by the rigourists to be hopeless rubbish, such as conjectures about sums of divergent series, was duly committed to the flames. 'Divergent series are' wrote Abel, 'the work of the devil'. They only cause 'calamities and paradoxicalities'. (...) The idea of a proof which deserves its name and still is not conclusive was alien to the rigourists.

(Lakatos, 2005, p. 137, footnotes are omitted)

Even though the above quote is taken from a discussion which is quite different than ours, it is still clear that Lakatos endorses the importance of contradictions for the increase of mathematical knowledge. The legitimate presence of such "paradoxicalities" do not collapse or trivialize the system. For Lakatosian methodology, under these circumstances, mathematicians still prove theorems - even sometimes with "proofs that do not prove" or with informal proofs. The existence of contradictions is therefore central for Lakatosian methodology to operate. At the end, contradictions perhaps are not included in the final theory for various metaphysical commitments that I shall not discuss here, yet, during the course of their development, the contradictions are appreciated and acknowledged, and perhaps even expected and desired in Lakatosian methodology. 
There can be suggested various ontological and epistemological reasons why contradictions, thus inconsistencies, are carefully excluded from the final theory. To the best of my knowledge, neither Lakatos nor Hintikka discusses the origins of their ontological commitment to classical Boolean logic, and the role of this commitment in their methodology in detail. Nevertheless, this commitment does not constitute an essential and unchangeable component of their methodology and research programs. The dialectic and discussive nature of their methodology necessarily requires an inconsistency-tolerant framework.

Now, going back to the similarities between Lakatosian and Hintikkan methodologies, one of the most important similarities between Lakatosian method and Hintikkan method becomes obvious after a brief look at the aforementioned road-map of Lakatosian methodology: Lakatosian methodology is also a selfcorrecting inquiry under the presence of inconsistencies. As we observed, for Hintikkan methodology that is an important aspect of an interrogative inquiry. For Lakatos, similarly, the process of mathematical discovery corrects itself by dealing with counter-examples, proofs that do not prove and similar anomalies and monsters. Lakatos goes further and introduces various methods for the self-correcting procedure. He employes three main strategies to implement the method of proofs and refutations: monster-barring, exception-barring and lemma incorporation (Başkent, 2012).

The method of monster-barring deals with the objects which are not in mind when the conjecture is first suggested. The method of exception-barring accepts that the theorem in its stated form is not valid due to the emergence of some genuine counterexamples targeting the correctness of the theorem itself. Lemma incorporation depicts the way we turn the counterexamples into new examples, and how those new examples are helpful for the modified and re-formulated version of the theorem. Note that even if these methods try to maintain a consistent and coherent logical system for the theory, in an a priori fashion they accept inconsistencies first, and go on with further deductions in a coherent way - this is what makes this system paraconsistent. Proofs and Refutations provides various cases and examples for Lakatosian reasoning with inconsistencies. In Proofs and Refutations various contradictory situations are discussed, solved, discussed again and resolved.

Now, Hintikka alludes to similar notions when he considers the Socratic method of elenchus: it is a dialogue, it is dialectic and there is a strategic component similar to Lakatos's. In Hintikka, the strategic and game theoretical elements are clearer and carefully underlined.

Another main requirement that can be addressed to the interrogative approach - and indeed to the theory of any goal-directed activity - is that it must do justice to the strategic aspects of inquiry. Among other things, it ought to be possible to distinguish the definitory rules of the activity in question from its strategic rules. The former spell out what is possible at each stage of the process. The latter express what actions are better and worse for the purpose of reaching the goals of the activity. This requirement can be handled most nat- 
urally by doing what Plato already did to the Socratic elenchus and by construing knowledge-seeking by questioning as a game that pits the questioner against the answerer. Then the study of the strategies of knowledge acquisition becomes another application of the mathematical theory of games, which perhaps ought to be called "strategy theory" rather than "game theory" in the first place. The distinction between the definitory rules - usually called simply the rules of the game - and strategic principles is built right into the structure of such games.

(Hintikka, 2007, p. 19)

The terminology and the context are different between the Hintikkan inquiry and the Lakatosian method. Yet, as the above quote illustrates, the strategic element is obvious in both. Additionally, there is another underlying tone of paraconsistency in elenchus, yet, in order to maintain our current focus, we will not dwell on this connection in this work (Carnielli, 2009).

Lakatosian and Hintikkan methods share various qualities including their reliance on inconsistency. Yet, I need to argue somehow more on their understanding of inconsistency. I will achieve it in the next section.

\section{Hintikka, Lakatos and the Inconsistent}

In another work, I argued that Hintikka's approach to inquiry in his interrogative models is misleading in excluding inconsistencies. I claimed that inconsistencies are epistemically central for knowledge increase in dynamic epistemic procedures such as dialogues and dialectics (Başkent, 2014).

A similar approach can be taken to analyze the Lakatosian methodology in the context of philosophy and methodology of mathematics. For this, we first need to remember the dialectical roots of Lakatosian method of proofs and refutations (PR, for short), and then the intrinsic relationship between dialectic and paraconsistency. In short, I will claim that Lakatosian methodology, via dialectic, is paraconsistent in nature - even though Lakatos himself did not make such a claim. Moreover, what renders Lakatosian philosophy paraconsistent also applies to Hintikkan method of inquiry. Let me now elaborate.

The relationship between PR and dialectic has been pointed out earlier by several authors (Kiss, 2006; Kvasz, 2002). For Lakatos, to improve the proof and the theorem, we need counter-examples and disproofs or proofs that do not prove.

Proofs that do not prove hint out an essential element of Lakatosian method of PR. For increase in knowledge, to improve the theorem and its proof, to revise the theory, we indeed rely on a proof that does not prove what it is set out to prove. In Lakatosian method, proofs are generally examined by raising counter-examples to them which in effect create a contradiction, thus an inconsistency. The proof is put forward, then after some quasi-emprical testing, some counter-examples are developed. At this moment of the method of PR, 
the method itself admits an inconsistency. Alas, PR chooses a strategy in which the proof, the proof that does not prove, is revised and improved. Granted, Lakatos strives to achieve consistency and coherence by his method. Any application of the method of proofs and refutations, with its negative and positive heuristics and protective belt, aims at a consistent and a coherent theory. I call this the meta-logical commitment of Lakatosian methodology. In other words, Lakatosian methodology is not committed to paraconsistency or dialetheism for that matter. Nevertheless, it needs inconsistencies to operate at the object level. They can be counter-examples, they can be various components of the theorem, their lemmata or their concepts which create an inconsistency. In Lakatosian methodology, when the proofs do not work as intended, it is not because of a simple error. Lakatos details them carefully in his work (Lakatos, 2005; Lakatos, 1979).

What the method of proofs and refutations suggests as a next step after coming across to inconsistencies is not a counter-argument to my claim that Lakatosian methodology is paraconsistent in essence. The reason is quite simple. The decision to revise the theory by using the method of proofs and refutations (and more importantly to determine the specific ways to achieve this revision based on the mathematical object theory at hand) is taken under the very existence of inconsistencies. I argued along these lines earlier.

Another way of looking at this issue is to investigate the dialectic roots of Lakatosian method. As mentioned in Corfield's outline of the method of proofs and refutations (see Chapter 2), the occurrence of counter-examples is an indispensable aspect of the method of PR. We can see the counter-examples as anti-theses where the initial proof attempts and immature theorems are the theses. Then, the Lakatosian dialectic operates and produces a synthesis using both thesis and anti-thesis. Lakatos himself often explicitly employs Hegelian method in his work as well (Lakatos, 2005, p. 145-6).

However, the very same Hegelian method is paraconsistent. The observation that dialectic is a paraconsistent methodology can be traced back to Hegel himself (Ficara, 2013; Kvasz, 2002; Priest, 1989). The core idea, as we already applied to Lakatosian methodology, is the fact that dialectic requires the presence of contradictory opinions, and operates under the very inconsistencies, yet produces a sound output. In this paper, I will not repeat the arguments in detail as to why dialectic can be considered as a dialetheic (and a paraconsistent) system. Yet, I will underline why dialectic, and in general dialogical systems are paraconsistent following Jaśkowski's argument for discussive logics (Jaśkowski, 1999). In a dialogue, assume that a player received two answers $p$ and $\neg p$ at different times. Nevertheless, it is completely possible that there exists a proposition $q$ which is nowhere true in the model. Thus, $q$ may not be deducible under the presence of a contradiction. Therefore, for some $p$ and $q$, we observe $p, \neg p \forall q$. Thus, the dialogue is paraconsistent. It does not entail that in all dialogues we have contradictory answers and a proposition that still does not follow. Yet, it means that the logic we use to formalize such systems should be in fact inconsistency-friendly. This is a call for extending the classical logic to an inconsistency-friendly, paraconsistent logic. In a paraconsistent logic, the 
classical logic can be a special case of the paraconsistent system, which serves our aim here. The argument we presented here for logical systems applies to dialectic and to logics that can describe dialectic reasoning as well, which after all applies to Hintikkan inquiry and Lakatosian method of PR. Notice that we are not describing a logic of dialectics here, instead, we use the fact that any formal system that uses dialectical reasoning intrinsically can descriptively be analyzed within a paraconsistent logical framework. Thus, it would not be wrong to claim that procedures and processes that use dialectical way of reasoning fit and embed in paraconsistent logic. In short, if the Lakatosian method has dialectic roots, and dialectic itself is paraconsistent in nature, then the method of proofs and refutations enjoys being a paraconsistent methodology. This argument (via dialectic) indirectly shows that Lakatosian method of PR is paraconsistent.

Another argumentation from paraconsistent logic can also be given (Priest \& Thomason, 2007). An intriguing aspect of paraconsistency is the view that it considers the "consistent" as a special case of the "inconsistent" as I briefly pointed out earlier.

The Euclidean conception of proof cannot characterise the history of mathematics. Lakatos' conception of proof as a fallible enterprise, starting from things that appear to be true, but which are subject to revision in the light of counter-examples, appears much more plausible. (...) Mathematicians and logicians are undoubtedly much more self-conscious about formulating the starting points, their axioms. But the axioms are no infallible epistemological bedrock. They are merely places where proof may stop, pro tem; they are still liable to be challenged by appropriate counter-examples. And this is just as true of the axioms of logic as those of mathematics. The development of paraconsistent logic can be seen as a clear case of this.

(Priest \& Thomason, 2007)

This line of thought constitutes another argument for the paraconsistency of Lakatosian methodology. Namely, even if its overall goal is to establish a consistent and coherent theory, proofs and refutations may admit inconsistencies, and the consistent case is merely a special case for the broader inconsistencytolerant framework of proofs and refutations.

This establishes that the Lakatosian method of proofs and refutations is inconsistency-tolerant and in fact paraconsistent.

So far, I have discussed the Lakatosian method of proofs and refutations (PR, for short) and its relations to paraconsistency. Now, I will argue that the same elements that render Lakatosian method paraconsistent applies to Hintikka's interrogative models of inquiry (IMI, for short) as well.

In order to achieve this, I will explicitly identify some of the common elements in PR and IMI that relate them to paraconsistency and dialetheia. 
- Both PR and IMI is about knowledge increase caused by (quasi-)empirical testing.

- When the empirical test produces a contradictory result, both PR and IMI has a constructive strategy to follow instead of rendering the model trivial, and resetting the procedure.

- Both PR and IMI have some erotetic aspects where questions themselves are central to the inquiry.

- Both PR and IMI are seen as activities.

Notice that the above list is not exhaustive and it can easily be applied to various other dialogical, erotetic and discussive systems.

Let us now elaborate more on those points.

Both PR and IMI is about knowledge increase caused by empirical testing In PR, testing the hypothesis is essential. In fact, this is the point where Lakatos's philosophy converges to empiricism. Lakatosian approach tests the hypothesis, experiments on it, produces counter-examples that are directed towards the theorems, the hypothesis or its concepts or definitions.

In IMI, the hypothesis or the initial question is tested by asking questions to the oracle or nature from whom the right answers are collected. It can be argued that the empirical aspects of IMI are not as strong as in PR. Yet, this line of criticism mistakenly considers IMI as an analytical method where questions essentially support the deductive procedure.

What distinguishes PR as a methodology in mathematics is its quasi-empirical aspects that diverge from analyticity. In IMI, on the other hand, Hintikka distinguishes two ways to increase knowledge. One is the deductive and analytical method based on the previous answers obtained in the inquiry and the rules of logic. Second, and the most important one for our purposes here, is the inquiry part where the inquirer poses questions to the nature or oracle. This breaks the chain of analyticity, and constitutes an empirical or quasi-empirical test. A rational inquirer would not ask analytical or deductive questions. He simply would ask the question for which he needs answers for. Therefore, those answers cannot be a part of his original theory.

I must emphasize that my understanding of "quasi-empiricisim" extends to formal sciences as well. In IMI, a question to the oracle constitutes a quasiempirical testing if the subject matter is a mathematical theorem or a theoretical physical result.

When the empirical test produces a contradictory result, both PR and IMI has a constructive strategy to follow The purpose of the (empirical or quasiempirical) experiments in PR and IMI is indeed to test the hypothesis. In some cases, the tests can produce some results that may contradict the hypothesis which is being tested. This is a perfectly routine modus operandi for PR and 
IMI. Namely, in questioning and in experimentation, the inquirer/tester can be wrong, and this is perfectly understandable and expectable. Yet, from a formal perspective, this creates, what I call, an instant contradiction. At that particular moment when the results of the tests are received, what we have is an inconsistent system. Yet, as we have emphasized throughout this paper, this contradiction does not render neither PR nor IMI trivial. In fact, both PR and IMI has a well-defined strategy to follow under such instant inconsistencies.

Both PR and IMI have some erotetic aspects Both PR and IMI posit a metaphysical stand when it comes to question generation. In PR, for example, it is not clear or precisely defined, how one can develop the right tests and quasiexperiments that can produce the clever counter-examples. Similarly, in IMI, it is not clear how the initial question(s) directed to the nature/oracle are formulated in the first place. Such ontological aspects of PR and IMI fall outside the domain of this paper, yet, both PR and IMI does not explain how those questions are generated. Question generation is what separates PR and IMI from analytical or purely deductive procedures. Notice that some of such questions -the ones that cause revisions or updates- cause inconsistencies. Thus, taken as a metaphysical and formal system, PR and IMI can produce those questions which create inconsistencies. This means that both are inconsistency-tolerant and paraconsistent.

Both PR and IMI are seen as activities Lakatos's emphasis on mathematics as a quasi-empirical science and an activity can be traced throughout Proofs and Refutations (Lakatos, 2005). The broader picture of the game of proofs and refutations points to an activity which is continuous, perhaps never ending process of constructing, deconstructing and reconstructing the concepts, theorems and proofs. From a dialectical perspective, PR being an activity is crucial as well. The activity continues, concepts are dialectically formed, and de-formed, and re-formed ad-infinitum. Moreover, mistakes happen, theorems are falsified, concepts are redefined. Activity also takes the form of quasi-experimentation as we already mentioned.

Now, let me elaborate how the features above appear in IMI, and render it inconsistency - tolerant.

First of all, I argue that IMI also conducts empirical testing as part of its methodology. IMI has two methods for knowledge increasing: deduction and questioning. In this paper, we leave the analytical discussions on deduction and knowledge increase aside, and focus on the questioning aspect of IMI. In IMI, questions, in fact, answers to those questions, introduce new elements to the inquiry, furthermore these questions/answers are the only way to introduce new information. It is an entirely different question how the answers and their data are processed, selected or omitted in an inquiry (Hintikka, 2007, p. 221). 
Moreover, it can also be argued that selecting the data just to maintain the consistency cannot be incorporated to interrogative inquiries (Başkent, 2014).

However, the question - answer procedure of inquiry contains empirical elements. Even if the way that the questions are generated requires a metaphysical commitment, the way that they are answered is empirical and a posteriori in a broad sense. Otherwise, epistemically and game theoretically, then questioning makes no sense - why would a rational agent ask a question whose answer does not have the potential to bring along new information or ask an irrelevant question? Clearly, our argument does not entail that all answers require such an empirical procedure. Yet, our thesis simply point out that question - answer protocol allows empirical testing, even if it may not necessitate it per se.

Second, I briefly discussed bracketing in Hintikka's IMI as a strategy to avoid contradictions. Either with bracketing, or instead without using bracketing and replacing it with some choice procedure, IMI functions with contradictions. Even if the end-result for Hintikka is ideally a consistent system without contradictions, the very existence of bracketing acknowledges their role, existence and emergence in IMI. Similar to Lakatos's various methods to maintain the consistency, Hintikkan IMI has its own slightly less sophisticated way of maintaining the consistency and coherence of its system.

Third, the erotetic aspects of question generation is a crucial point of both IMI and PR. However, Hintikka himself does not say much about it when it comes to IMI. Yet, we believe, question generation in IMI is directly related to rationality of the inquirer. Clearly, the inquirer can raise any questions that the oracle can answer with yes/no answers. This does not rule out that the inquirer shall direct trivial or analytical questions to the oracle. What restricts the inquirer from asking trivial questions is the rationality element of the player, the inquirer. Assuming that he is committed to winning the game of inquiry, the inquirer will try to ask relevant and non-trivial questions, and try to maximize his gain from the questions. Ideally, he will receive consistent and coherent answers. However, in reality, in an empirical or computationally challenging inquiry, the inquirer can receive contradictory answers, and it is perfectly normal. Similar to Jaśkowski's argument we mentioned earlier, IMI admits inconsistencies (Başkent, 2014).

Hintikka also discusses the probabilistic aspects of the question-answer activity of IMI (Hintikka, 1987). This portrays a bit more realistic picture of IMI, and in a different way underlines the role of questions in IMI.

Finally, as Garrison also emphasized, Hintikkan IMI has some similarities as an activity to Laudan and Lakatos (Garrison, 1988). It can be argued that in Hintikka, the activity aspect of the process can be most easily seen in the question formation. After all, the deduction is straight-forwardly defined, and the only creative room in the process is the activity of asking and forming questions. This creativity can perhaps be overshadowed by a know-it-all oracle, and this most certainly shortens the period of the activity. Instead of experimentation and various back-and-forth questioning, the oracle -ideally- produces the correct answer immediately and instantly. Nevertheless, this procedure renders still IMI as a dialog and an activity. 
Notice that the activity aspect of Lakatosian PR is much more evident than that of Hintikkan IMI, and in fact the process of PR relies on the quasi-empirical activity as the generator of counter-examples. Yet, knowledge generation as an activity is a quite broad approach to various formalisms, and what I have tried to accomplish in this paper can be considered very similar to Garrison's attempt to unite Hintikkan IMI with Laudan's conception of science as a problem-solving, and question-answering activity. (Garrison, 1988).

In conclusion, I argued that Lakatosian PR and Hintikkan IMI share various aspects that render both inconsistency-tolerant frameworks.

\section{Conclusion}

Lakatos's and Hintikka's methods differ on a variety of points. Yet, within the scope of this paper, they are united on their approach to the inconsistent. However, I argued that their reading of the inconsistent, within their own goals and framework, is misleading, even if Hintikka later showed some interest towards paraconsistency. In fact, both PR and IMI rely heavily on the existence of (perhaps temporary) inconsistencies and contradictions.

The role of dialectics both in Hintikka and Lakatos is an interesting direction to pursue, and we restricted ourselves to briefly touching to that issue. Much more can be said, and especially in Lakatosian case studies, a more detailed outline of Lakatosian dialectic can be given within a broader framework which goes beyond the limits of a single research paper.

Also, more importantly, philosophers change their opinions and they revise their ideas - sometimes paraconsistently, sometimes classically perhaps. So did Hintikka. In (Hintikka, 2009), the Hintikka we read is quite different than what is represented in this paper as he considers (even remotely) the possibility of combining IF logic with paraconsistent logics to create a common framework.

Finally, the ideas we presented in this paper can easily extend to broader issues in philosophy of mathematics suggesting a paraconsistent view of the subject. We leave such investigations to a future work.

Acknowledgements I acknowledge the suggestions of the two anonymous referees. The paper has benefited a lot from the feedback of Marco Panza.

\section{References}

BaşKent, Can. 2011. A Logic for Strategy Updates. Pages 382-3 of: van Ditmarsch, HANS, \& LANG, Jerome (eds), Proceedings of the Third International Workshop on Logic, Rationality and Interaction (LORI-3), vol. LNCS 6953.

BAŞKEnT, CAn. 2012. A Formal Approach to Lakatosian Heuristics. Logique et Analyse, 55(217), 23-46. 
BAŞKENT, CAN. 2014. Towards Paraconsistent Inquiry. under review.

BaşKent, CAn, \& BaG̃ÇE, SAmet. 2009. An Examination of Counterexamples in Proofs and Refutations. Philosophia Scientiae, 13(2), 3-20.

Carnielli, Walter A. 2009. Meeting Hintikka's Challenge to Paraconsistentism. Principia, 13(3), 283-97.

Carnielli, Walter A., Coniglio, M. E., \& Marcos, J. 2007. Logics of formal inconsistency. Pages 15-107 of: GabBay, Dov, \& Guenthner, F. (eds), Handbook of Philosophical Logic, vol. 14. Springer.

CoRfield, DAvid. 1997. Assaying Lakatos's History and Philosophy of Science. Studies in History and Philosophy of Science, 28(1), 99-121.

FiCARA, ElEnA. 2013. Dialectic and Dialetheism. History and Philosophy of Logic, 34(1), 35-52.

GARRISON, JAMEs W. 1988. Hintikka, Laudan and Newton: An Interrogative Model of Scientific Inquiry. Synthese, 74, 145-171.

Genot, Emmanuel J. 2009. The Game of Inquiry: The Interrogative Approach to Inquiry and Belief Revision Theory. Synthese, 171(2), 271-289.

Halonen, Ilpo, \& HintikKa, JaAkKo. 2005. Toward a Theory of the Process of Explanation. Synthese, 143(1), 5-61.

HintikKA, JAAKKo. 1962. Knowledge and Belief. Cornell University Press.

HinTIKKA, JAAKKO. 1984. The Logic of Science as a Model-Oriented Logic. Pages 177-185 of: PSA: Proceedings of the Biennial Meeting of the Philosophy of Science Association, vol. 1. The University of Chicago Press.

HINTIKKA, JAAKKO. 1987. The Interrogative Approach to Inquiry and Probabilistic Inference. Erkenntnis, 26, 429-442.

HINTIKKA, JAAKKO. 1988. What is the Logic of Experimental Inquiry? Synthese, 74, 173-190.

HintikKA, JAAKKo. 2007. Socratic Epistemology. Cambridge University Press.

HintikKA, JAAKKO. 2009. IF Logic Meets Paraconsistent Logic. Pages 3-13 of: Carnielli, Walter A., Coniglio, M. E., \& D’Ottaviano, I. M. L. (eds), The Many Sides of Logic. College Publications.

HintikKa, JAAKKo, \& HARRIS, STEPHEN. 1988. On the Logic of Interrogative Inquiry. Pages 233-240 of: PSA: Proceedings of the Biennial Meeting of the Philosophy of Science Association, vol. 1. The University of Chicago Press.

Hintikka, JaAkKo, Halonen, Ilpo, \& Mutanen, Arto. 1999. Interrogative Logic as a General Theory of Reasoning. Pages 295-337 of: ET AL, Dov GabBAy (ed), Studies in Logic and Practical Reasoning, vol. 1. Kluwer. 
JAŚKOWSKI, STANISŁAW. 1999. A Propositional Calculus for Inconsistent Deductive Systems. Logic and Logical Philosophy, 7(1), 35-56.

KISS, OlgA. 2006. Heuristics, Methodology or Logic of Discovery? Lakatos on Patterns of Thinking. Perspectives on Science, 14, 302-317.

Koetsier, Teun. 1991. Lakatos' Philosophy of Mathematics: A Historical Approach. North-Holland.

Kvasz, L. 2002. Lakatos' Methodology Between Logic and Dialectic. In: KAmPIS, G., Kvasz, L., \& StÖlzner, M. (eds), Appraising Lakatos: Mathematics, Methodology and the Man,. Kluwer.

LaKatos, Imre. 1979. Mathematics, Science and Epistemology. Cambridge University Press.

Lakatos, Imre. 2005. Proofs and Refutations. Cambridge University Press.

Priest, Graham. 1989. Dialectic and Dialetheic. Science \& Society, 53(4), $388-415$.

Priest, Graham, \& Thomason, Neil. 2007. 60\% Proof - Lakatos, Proof and Paraconsistency. Australasian Journal of Logic, 5, 89-100.

Rahman, Shahid, \& Carnielli, Walter A. 2000. The Dialogical Approach to Paraconsistency. Synthese, 125, 201-231.

Rahman, Shahid, \& Tulenheimo, Tero. 2009. From Games to Dialogues and Back. Pages 153-208 of: Maher, Ondrej, Pietarinen, Ahti, \& TuLenheimo, Tero (eds), Games: Unifying Logic, Language and Philosophy. Springer. 